\title{
Lost expression of ADAMTS5 protein associates with progression and poor prognosis of hepatocellular carcinoma
}

This article was published in the following Dove Press journal:

Drug Design, Development and Therapy

24 March 2015

Number of times this article has been viewed

\author{
Chongyi Li, ${ }^{1,2}$ \\ Yanli Xiong' \\ Xueqin Yang' \\ Lin'ang Wang' \\ Shiheng Zhang' \\ Nan Dai' \\ Mengxia $\mathrm{Li}^{\prime}$ \\ Tao Ren' \\ Yuxin Yang' \\ Shu-Feng Zhou ${ }^{3}$ \\ Lixia $\mathrm{Gan}^{2}$ \\ Dong Wang' \\ 'Cancer Center, Daping Hospital \\ and Research Institute of Surgery, \\ ${ }^{2}$ Department of Biochemistry \\ and Molecular Biology, \\ The Third Military Medical \\ University, Chongqing, People's \\ Republic of China; ${ }^{3}$ Department \\ of Pharmaceutical Sciences, College \\ of Pharmacy, University of South \\ Florida, Tampa, FL, USA
}

Correspondence: Dong Wang

Cancer Center, Daping Hospital

and Research Institute of Surgery,

The Third Military Medical University,

10 Changjiang Zhi Road, Yuzhong

District, Chongqing 400042, People's

Republic of China

Tel +86 236875715 I

Fax +862368894062

Email dongwang64@hotmail.com

Lixia Gan

Department of Biochemistry

and Molecular Biology, The Third Military

Medical University, 30 Gaotanyan

Main Street, Shapingba District, Chongqing

400038, People's Republic of China

Tel +862368752945

Fax +86236889 4062

Email ganlixia@yahoo.com
Abstract: Altered expression of ADAMTS5 is associated with human carcinogenesis and tumor progression. However, the role of ADAMTS5 in hepatocellular carcinoma (HCC) is unclear. This study analyzed ADAMTS5 expression in HCC tissues and tested for association with clinicopathological and survival data from HCC patients and then explored the role of ADAMTS5 in HCC cells in vitro. Paraffin blocks from 48 HCC patients were used to detect ADAMTS5 and vascular endothelial growth factor (VEGF) expression and microvessel density (MVD). A normal liver cell line and HCC cell lines were used to detect ADAMTS5 expression and for ADAMTS5 manipulation. ADAMTS5 cDNA was stably transfected into HCC cells and ADAMTS5 expression assessed by Western blot analysis. Tumor cell-conditioned growth medium was used to assess human umbilical vein endothelial cell migration and Matrigel tube formation. Xenograft assay was performed to determine the role of ADAMTS5 in vivo. The data showed that the expression of ADAMTS5 was reduced in HCC, which was inversely associated with VEGF expression, MVD, and tumor size and associated with poor overall survival of HCC patients. Lentivirus-mediated ADAMTS5 expression significantly inhibited tumor angiogenesis by downregulating in vitro expression of VEGF and inhibiting migration and tube formations, and also inhibited tumor growth and VEGF expression and reduced MVD in vivo in a mouse xenograft model. Taken together, these results suggest that ADAMTS5 plays a role in suppression of HCC progression, which could be further studied as a promising novel therapeutic target and a potential prognostic marker in HCC.

Keywords: A disintegrin and metalloproteinase with thrombospondin motifs 5, tumor angiogenesis, tumor cell xenograft

\section{Introduction}

Liver cancer is the fifth most frequently diagnosed cancer and the second most frequent cause of cancer death in men in the world, while it is the seventh most commonly diagnosed female cancer and the sixth leading cause of cancer death in women. ${ }^{1}$ Liver cancer accounted for 748,300 new cancer cases and 695,900 cancer deaths in the world in 2008. Of these, approximately 50\% of liver cancer cases and deaths was estimated to occur in People's Republic of China. ${ }^{2}$ Hepatocellular carcinoma (HCC) represents $70 \%-85 \%$ of total liver cancer cases. Chronic hepatitis B virus or hepatitis $\mathrm{C}$ virus infection is the most frequent influential risk factor for $\mathrm{HCC}$ development, accounting for more than $50 \%$ of all HCC cases. ${ }^{2}$ To date, surgical resection and liver transplantation are the most effective therapies for HCC patients, but prognosis remains poor as a result of diagnosis at later stages of the disease and tumor reoccurrence (such as invasion and metastasis). ${ }^{2}$ Therefore, there is an urgent need to better understand the molecular characteristics and underlying biological mechanism, which may in turn provide 
novel strategies in prevention, early detection, treatment, and prediction of prognosis and treatment responses of this deadly disease. Recently, it has been reported that expression of the A disintegrin-like and metalloproteinase with thrombospondin motifs 5 (ADAMTS5) was reduced or lost in HCC cell lines. ${ }^{3}$ This observation needs to be confirmed in HCC and the role of ADAMTS5 in HCC requires definition.

Specifically, ADAMTSs are a family of proteins that function as proteoglycanases and share several distinct protein modules, including a propeptide region, a metalloproteinase domain, a disintegrin-like domain, and a thrombospondin type 1 motifs. ${ }^{4}$ Among ADAMTSs, ADAMTS5 is well known for its important role in cartilage biology, and knockout mouse studies showed that ADAMTS5 deletion was resistant to cartilage destruction $^{5}$ and that ADAMTS5 is the major aggrecanase in mouse cartilage in a mouse model of inflammatory arthritis. ${ }^{6}$ More recently, increasing evidence suggests a potential role of ADAMTS5 in cancer development and progression. For example, a previous study has shown that ADAMTS5 expression was downregulated in human breast cancer. ${ }^{7}$ ADAMTS5 gene was also hypermethylated in colorectal cancer and T-cell acute lymphoblastic leukemia. ${ }^{8,9}$ However, in contrast, in other reports, ADAMTS5 expression was shown to be upregulated in human glioblastoma compared to the normal brain tissues and promoted glioma cell invasiveness in in vitro culture. $^{10,11}$ These data suggest that ADAMTS5 may have different functions in different types of human cancer. Thus, the roles of ADAMTS5 in human cancers, including HCC, need to be further elucidated. Thus, in this study, we evaluated the expression of ADAMTS5 protein in a normal liver cell line and six HCC cell lines using Western blotting. We then analyzed ADAMTS5 expression and associated ADAMTS5 expression with clinicopathological data from patients as well as with vascular endothelial growth factor (VEGF) expression and microvessel density (MVD). After that, we explored the role of ADAMTS5 in HCC progression in vitro.

\section{Materials and methods}

\section{Patients and tissues}

Formalin-fixed paraffin-embedded HCC tissues and the surrounding non-tumor liver tissues were collected from 43 men and five women (age, 24-73 years; mean \pm standard deviation, $54 \pm 11$ years) who underwent liver resection in the Daping Hospital, Chongqing, People's Republic of China, between 2009 and 2010. Survival data were collected for all the patients and 41 of 48 patients $(85.4 \%)$ died of cancer metastasis or local recurrence. All patient data are shown in Table 1. In brief, all patients underwent surgery, and no
Table I Association of ADAMTS5 expression with clinicopathological factors in $48 \mathrm{HCC}$ patients

\begin{tabular}{|c|c|c|c|c|}
\hline Characteristics & Number of cases & Positive & Negative & $P$-value \\
\hline \multicolumn{5}{|l|}{ Age (years) } \\
\hline$<60$ & 32 & 16 & 16 & \\
\hline$\geq 60$ & 16 & 8 & 8 & $1.000^{\mathrm{a}}$ \\
\hline \multicolumn{5}{|l|}{ Sex } \\
\hline Male & 43 & 21 & 22 & \\
\hline Female & 5 & 3 & 2 & $1.000^{\mathrm{b}}$ \\
\hline \multicolumn{5}{|c|}{ Tumor degree of differentiation } \\
\hline Well & 6 & 2 & 4 & \\
\hline Moderate & 30 & 16 & 14 & \\
\hline Poor & 12 & 6 & 6 & $0.770^{\mathrm{b}}$ \\
\hline \multicolumn{5}{|l|}{ Stage } \\
\hline$I+I I$ & 26 & 18 & 8 & \\
\hline$I I I+I V$ & 22 & 6 & 16 & $0.004^{a}$ \\
\hline \multicolumn{5}{|l|}{ HBV } \\
\hline Positive & 38 & 18 & 20 & \\
\hline Negative & 10 & 6 & 4 & $0.477^{\mathrm{b}}$ \\
\hline \multicolumn{5}{|l|}{$\operatorname{AFP}(\mathrm{ng} / \mathrm{mL})$} \\
\hline$<20$ & 16 & 7 & 9 & \\
\hline$>20$ & 32 & 17 & 15 & $0.095^{\mathrm{a}}$ \\
\hline \multicolumn{2}{|l|}{ HCC tissue } & \multicolumn{3}{|c|}{ Adjacent tissue } \\
\hline Positive & & 21 & 3 & \\
\hline Negative & & 18 & 6 & $0.00 I^{c}$ \\
\hline
\end{tabular}

Notes: aChi-square test; 'Fisher's exact probability test; 'McNemar's test. Abbreviations: AFP, alpha fetoprotein; $\mathrm{HBV}$, hepatitis $B$ virus; $\mathrm{HCC}$, hepatocellular carcinoma.

patient had received chemotherapy or radiation. Tumor stage was classified according to the American Joint Committee on Cancer tumor-node-metastasis (TNM) classification. This study was approved by the Research Ethics Boards of the Daping Hospital and Research Institute of Surgery, and informed consent was obtained from all patients.

\section{Construction of tissue microarray and immunohistochemistry}

Paraffin blocks from $\mathrm{HCC}$ and normal tissues were constructed into tissue microarray (TMA) and reported previously. ${ }^{12}$ TMA blocks were cut into $4.5 \mu \mathrm{M}$ sections. TMA sections were stained for ADAMTS5 expression using a rabbit polyclonal antibody against ADAMTS5 at a dilution of 1:100 (\#ab41037; Abcam, Cambridge, UK), for VEGF using a mouse monoclonal antibody against VEGF (MAIXIN-BIO, Fuzhou, People's Republic of China), and for microvessels using a rabbit monoclonal antibody against CD34 at a dilution of 1:400 (\#ab81289; Abcam) according to a previous study. ${ }^{10}$ The color reaction was performed by using 3,3'-diaminobenzidine solution and the sections were counterstained with hematoxylin. Immunostaining was reviewed and scored by two investigators for percentage of 
cell staining and intensity of staining. All tissues were scored as 0 (negative), 1 (weak), 2 (strong), or 3 (very strong). The expression was considered negative if the final score was $0-1$, while it was considered positive if the final score was 2-3. MVD was defined as all CD34-positive endothelial cell(s) separate from adjacent microvessels according to a previously published study. ${ }^{13}$ MVD was quantified following visualization of microvessels at $200 \times$ magnification recorded by rotating the graticule to the view of the eyepiece to reveal the maximum number of stained vessels. For each section, three fields were captured and the results were expressed as the mean \pm standard deviation. The primary antibody was substituted with phosphate-buffered saline (PBS) as a negative control.

\section{Cell culture and reagents}

A normal liver cell line L02 and six human hepatomaderived cell lines (HepG2, SMMC-7721, Hep3B, PLC/ $\mathrm{PRF} / 5, \mathrm{MHCC} 97 \mathrm{~L}$, and MHCC97H) were obtained from Liver Cancer Institute, Zhongshan Hospital (Shanghai, People's Republic of China). Among these six HCC cell lines, MHCC97L and MHCC97H were established from the same parental HCC cell line and had a progressively increasing metastatic potential according to data published in previous studies. ${ }^{14}$ Hep3B and HepG2 cell lines had a low metastatic potential. These cell lines were cultured in Dulbecco's Modified Eagle's Medium (DMEM) (Thermo Fisher Scientific, Waltham, MA, USA) with $10 \%$ (v/v) fetal bovine serum (Hyclone, Logan, UT, USA), $100 \mathrm{U} / \mathrm{mL}$ penicillin, and $100 \mathrm{U} / \mathrm{mL}$ streptomycin in a humidified $5 \% \mathrm{CO}_{2}$ incubator at $37^{\circ} \mathrm{C}$. Recombinant VEGF was purchased from Sigma-Aldrich Co. (St Louis, MO, USA).

\section{Protein extraction and Western blot}

The level of intracellular ADAMTS5 was determined by Western blot as described previously. ${ }^{3,15}$ Equal protein aliquots from each sample were resolved in $8 \%$ sodium dodecyl sulfate-polyacrylamide gel electrophoresis gel and transferred onto a polyvinylidene fluoride membrane. After being blocked with $10 \%$ skimmed milk solution in PBS, the membranes were probed with an anti-ADAMTS5 antibody (diluted at 1:250 [\#ab41037; Abcam]) or an anti-ACTB antibody at a dilution of 1:2,000 (\#sc-47778; Santa Cruz Biotechnology Inc., Dallas, TX, USA). The membranes were then incubated with the horseradish peroxidase-labeled secondary antibody and the blots were detected by enhanced chemiluminescence solution Western blotting kits (Pierce, Rockford, IL, USA) according to the manufacturer's instructions.
Expression of $\beta$-actin served as a loading control. The density of the bands was quantified using Image $J$ software (National Institute of Health, Bethesda, MD, USA).

\section{Generation of stable ADAMTS5- expressed HCC sublines}

Human ADAMTS5 cDNA in pCMV6-XL4 vector was obtained from Origene (Rockville, MD, USA) and was used as a template to obtain full-length ADAMTS5 for construction of pLentis-CMV-ADAMTS5-PGK-PURO plasmid (the empty vector was obtained from Chongqing Lentis Bioscience, Chongqing, People's Republic of China). Lentivirus was then produced by Chongqing Lentis Bioscience and used to establish stable ADAMTS5-expressed Hep3B cells (Hep3B/TS5 or TS5). The control cells (Hep3B/Vec or Vec) were generated by using lentivirus carrying the corresponding empty vector. Stable cell lines were established by selection with $5 \mu \mathrm{g} / \mathrm{mL}$ puromycin.

\section{Preparation of the tumor cell culture-conditioned medium}

To prepare the tumor cell culture-conditioned medium (TCM) Hep3B/TS5 cells and Hep3B/Vec cells were seeded in six-well plates at a density of $1 \times 10^{5}$ and grown for 24 hours. Next, the cells were washed with PBS three times, and then re-cultured in $1 \mathrm{~mL}$ of serum-free media for an additional 24 hours. After that, the growth medium was collected and centrifuged at $12,000 \times g$ for 10 minutes at $4^{\circ} \mathrm{C}$. The supernatant (TCM) was stored at $-80^{\circ} \mathrm{C}$ until use.

\section{ELISA detection of VEGF levels in TCM}

Levels of VEGF concentrations in the TCM were quantified using a VEGF enzyme-linked immunosorbent assay (ELISA) kit (Thermo Fisher Scientific) according to the manufacturer's instructions.

\section{Human umbilical vein endothelial cell migration assay}

A Transwell system was used to detect human umbilical vein endothelial cell (HUVEC) migration according to a previous study, with some modifications. ${ }^{13}$ Briefly, confluent HUVECs were trypsinized and resuspended in serum-free DMEM. Cell suspensions containing 80,000 HUVECs were seeded into the upper chamber of Transwell inserts ( $8 \mu \mathrm{m}$ pore size; BD Biosciences, San Jose, CA, USA). The lower compartment was filled with various amounts of TCM. The cells were cultured for 16 hours at $37^{\circ} \mathrm{C}$. After that, the non-migrated cells were removed from the upper surface by scraping with 
a cotton swab, while cells that had migrated were stained with crystal violet, and the number of migrating cells per field was counted for the number of migrating cells in five random fields at $200 \times$ magnification.

\section{HUVEC Matrigel tube formation assay}

This assay was performed as described previously. ${ }^{16,17}$ In brief, HUVECs were seeded into a 24-well plate precoated with $400 \mu \mathrm{L}$ of thick Matrigel (BD) at a density of 128,000 cells per well and cultured in $800 \mu \mathrm{L}$ of TCM at $37^{\circ} \mathrm{C}$ and $5 \% \mathrm{CO}_{2}$ for 24 hours. The capillary tube formation was photographed using a Leica Microsystems DM IL phase contrast inverted microscope (Wetzlar, Germany) in five random fields at $100 \times$ magnification. Tubulogenesis was evaluated by counting the number of completely enclosed endothelial networks in each well. Each experiment was performed in triplicate and repeated once.

\section{Tumor cell growth and angiogenesis in xenograft assay and \\ immunohistochemical analysis}

All experimental procedures involving animal use were approved by the Ethics Committee of Daping Hospital and Research Institute of Surgery. Four-week old female BALB/c nude mice were purchased from the Shanghai Experimental Animal Center, Chinese Academy of Sciences (Shanghai, People's Republic of China). The experiments were performed according to previous studies. ${ }^{18,19}$ In brief, $1 \times 10^{7} \mathrm{Hep} 3 \mathrm{~B} / \mathrm{TS} 5$ or Hep3B/Vec cells in a volume of $200 \mu \mathrm{L}$ were injected subcutaneously into the right flank of mice. The body weight and tumor volume of each mouse were recorded weekly. After 35 days, mice were sacrificed and tumor xenografts were removed and fixed in a buffered formalin solution for immunohistochemical analysis of expression of ADAMTS5, VEGF, and CD34 as described in the construction of tissue microarray and immunohistochemistry. The results of immunohistochemistry were quantified by sum integral optical density using Image-Pro Plus analysis software. The tumor volume was calculated according to the formula $\mathrm{A} \times \mathrm{B}^{2} \times 0.52,{ }^{20}$ where A was the largest diam1 and B was the smallest diameter $(\mathrm{mm})$. The results were quantified by sum integral optical density using Image-Pro Plus analysis software.

\section{Statistical analysis}

Student's $t$-test was used to compare the expression of ADAMTS5 among different cell lines. McNemar's test was used to compare ADAMTS5 expression between HCC and distant paracarcinomatous tissues. The chi-square test or Fisher's exact probability test was conducted to evaluate the association of ADAMTS5 expression with clinicopathological characteristics. Spearman's rank correlation coefficient was used to assess association of ADAMTS5, VEGF, MVD, and tumor size. Kaplan-Meier curves and the log-rank test were used to assess the association of ADAMTS5 expression with overall survival (OS) of patients. Cox proportional hazards models were used to adjust for clinical factors. All statistical analyses were performed at the $P=0.05$ level using SPSS software version 19.0 (IBM Corporation, Armonk, NY, USA).

\section{Results}

\section{Expression of ADAMTS5 in liver cell lines} In this study, we first assessed expression of ADAMTS5 protein in a normal liver cell line and six HCC cell lines. Western blot data showed that ADAMTS5 zymogen (120 kDa) was the major expression form, but other forms of ADAMTS5 were also expressed at a very low level. Specifically, the normal liver L02 cells had a higher level of ADAMTS5 expression compared to the other six $\mathrm{HCC}$ cell lines (HepG2, SMMC-7721, Hep3B, PLC/PRF/5, MHCC97L, and $\mathrm{MHCC} 97 \mathrm{H})$ (Figure 1).

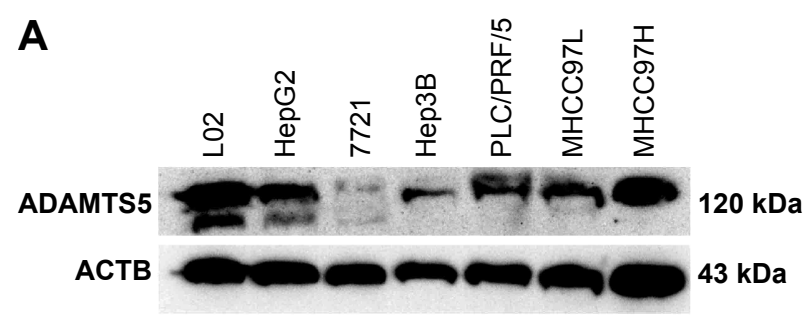

B

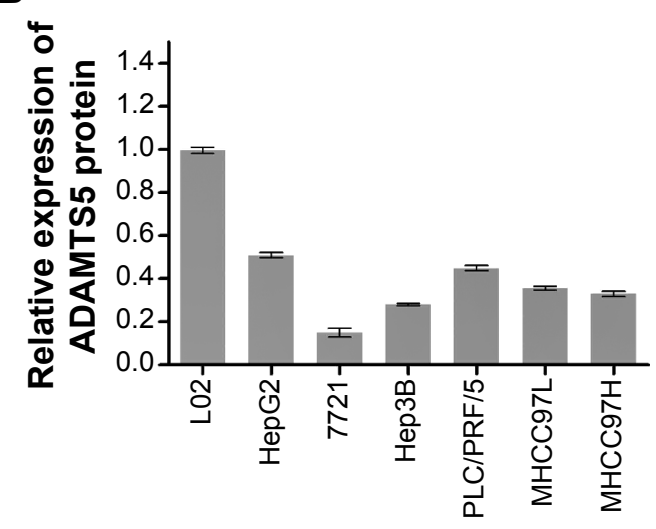

Figure I Expression level of ADAMTS5 protein in liver hepatocellular carcinoma cell lines.

Notes: (A) Cell lysates of normal liver cell LO2 and size hepatocellular carcinoma cell lines (HepG2, SMMC-772I, Hep3B, PLC/PRF/5, MHCC97L, and MHCC97H) were subjected to Western blot analysis of ADAMTS5 expression and re-probed with the $\beta$-actin antibody as a loading control. (B) The graph summarizes WB data $(n=3)$. WB is the relative protein level of ADAMTS5. 


\section{Differential expression of ADAMTS5 in $\mathrm{HCC}$ and paracarcinomatous tissues}

To determine ADAMTS5 expression in HCC specimens, we performed immunohistochemical analysis and found that ADAMTS5 protein was primarily expressed in the cytoplasm. The positive staining rate of ADAMTS5 antibody was $50 \%$ (24/48) in HCC tissues, while it was $81.3 \%$ $(39 / 48)$ in the adjacent paracarcinomatous tissues. The statistical analysis showed that ADAMTS5 expression was significantly lower in HCC tissues versus paracarcinomatous tissues $(P<0.05$ using McNemar's test). Representative immunohistochemical staining of ADAMTS5 protein in HCC tissue is shown in Figure 2.

\section{Association of ADAMTS5 with VEGF expression and MVD in HCC tissues}

Angiogenesis plays an important role in HCC development and a previous study demonstrated that ADAMTS5 was able to inhibit tumorigenesis through suppression of tumor angiogenesis and proangiogenic factor VEGF expression. ${ }^{21}$ We thus assessed VEGF expression and MVD using an anti-VEGF and anti-CD34 antibody in HCC tissue samples, respectively. The data showed that the MVD value ranged from six to 98 microvessels per $200 \times$ field (median, 39) (Figure 2) in HCC tumor tissues. VEGF was mainly expressed in the cytoplasm of tumor cells (Figure 2) in $45.8 \%(22 / 48)$ of HCC samples. We then compared ADAMTS5 expression with VEGF levels and MVD using a Spearman's rank correlation test and found that they were significantly associated (MVD, $r=-0.530, P<0.001$; VEGF, $r=-0.640, P<0.001)$. ADAMTS5 expression was also inversely associated with tumor size $(r=-0.361, P=0.012)$ (Table 2).

\section{Association of ADAMTS5 expression with survival of $\mathrm{HCC}$ patients}

The association of ADAMTS5 expression with survival of HCC patients was assessed using Kaplan-Meier curves. The results showed that patients with highly ADAMTS5-expressed HCC had remarkably better OS after curative hepatectomy compared to those without or with low ADAMTS5-expressed HCC (29.56 \pm 4.27 versus $12.96 \pm 1.93$ months; $P<0.001$ ) (Figure 3). Similarly, VEGF expression, high MVD, and larger HCC size were also associated with poor OS (Figure 3).

Univariate analysis showed that ADAMTS5 and VEGF expression, MVD, and HCC size and stage were significantly associated with $\mathrm{HCC}$ prognosis $(P<0.05)$ (Table 3 ). Multivariate analysis calculated by Cox regression showed that ADAMTS5 expression was an independent prognostic factor for OS of HCC patients after curative hepatectomy $(P<0.05)$ (Table 3).

\section{Effect of tumor cell-expressed ADAMTS5 on reduction of HUVEC migration and capillary tube formation}

To determine the role of ADAMTS5 in the progression of HCC, we first established a stably ADAMTS5-expressed HCC cell line and assessed effects of ADAMTS5 on angiogenesis in vitro. As shown in Figure 4A and B, ADAMTS5 expression significantly inhibited VEGF level $(P<0.001)$. Moreover, the number of migrated HUVECs was significantly smaller in the ADAMTS5 group compared to the control group $(P<0.01)$; however, the reduced HUVEC migration capacity was largely restored by addition of recombinant VEGF $(P<0.01)$ (Figure $4 \mathrm{C}$ and $\mathrm{D})$. Matrigel tube formation assays were performed to assess the effect of ADAMTS5 on the capillary tube formation ability of HUVECs and showed that there were fewer capillary tubes formed in the presence of TCM derived from HCC cells with ectopic ADAMTS5 expression compared to that of the control cells $(P<0.01)$, and this was reversed by addition of recombinant VEGF $(P<0.01)$ (Figure 4E and F). These data suggest that ADAMTS5 negatively regulates the capacity of tumor cell-promoted HUVEC migration and capillary tube formation by downregulation of VEGF expression in vitro.

\section{Effect of ADAMTS5 on tumor growth and angiogenesis in vivo}

To further demonstrate the role of ADAMTS5 in tumor angiogenesis and growth in vivo, we performed a BALB/c mouse hepatoma xenograft assay. The data showed that tumor xenografts generated with Hep3B/TS5 cells grew more slowly than those generated with Hep3B/TS5 cells $(P<0.01)$ (Figure 5A). Hep3B/TS5 cell xenografts weighed less than Hep3B/Vec cell xenografts $(P<0.0001)$ (Figure 5B).

Tumor sections were analyzed using immunohistochemistry, and representative images are shown in Figure 5C. As expected, tumor xenografts from the Hep3B/TS5 cells had lower expression of VEGF. The MVD detected using antiCD34 antibody was significantly lower in the Hep3B/TS5 group than in the Hep3B/Vec group $(P<0.01)$ (Figure 5D), which correlated well with the tumor growth rate shown in Figure 5A and B. Together, these results indicate that ADAMTS5 significantly inhibited tumor growth and angiogenesis in vivo by downregulating VEGF expression. 

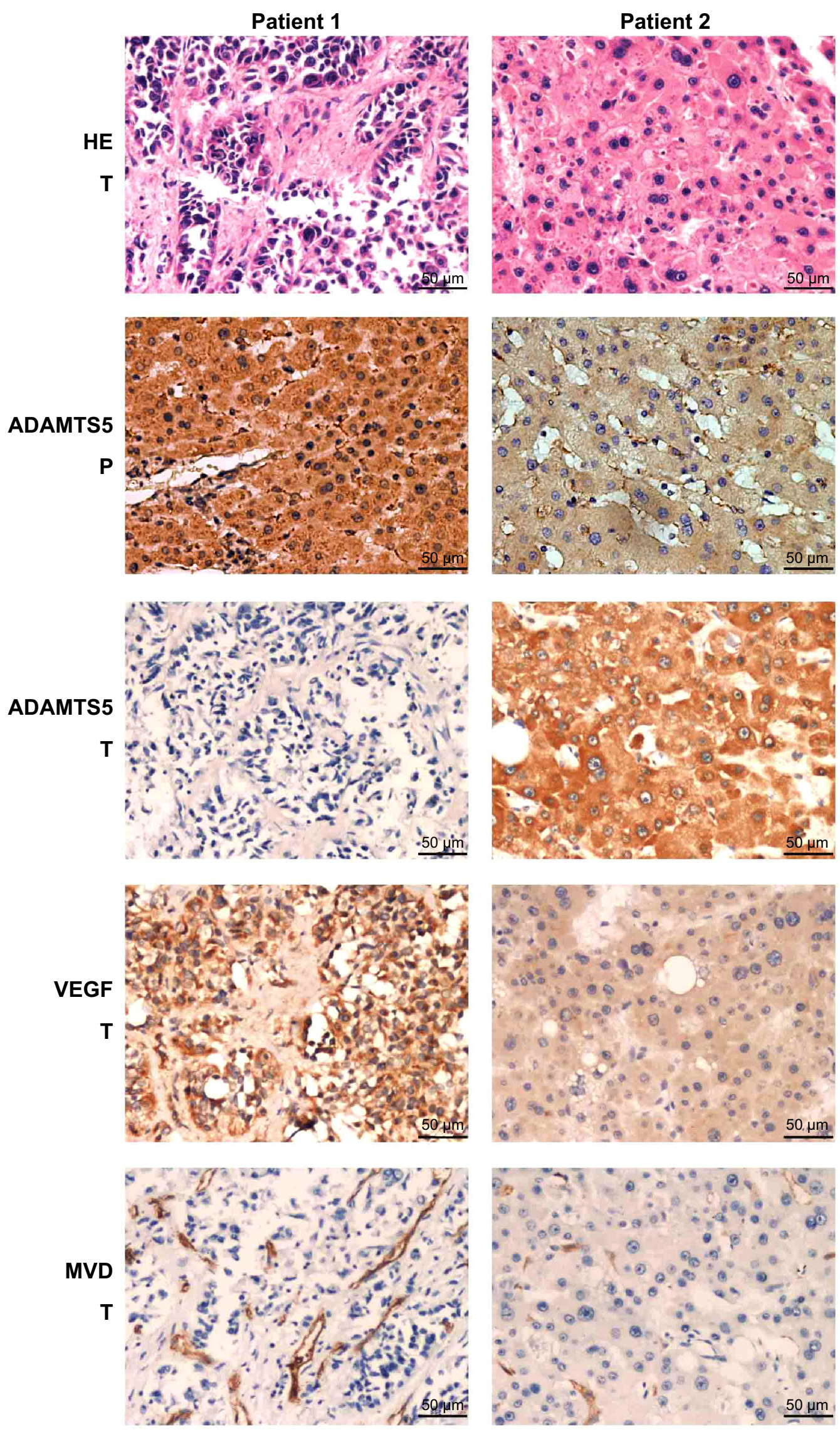

Figure 2 Immunohistochemical staining of ADAMTS5, VEGF, and CD34 in hepatocellular carcinoma tissues.

Abbreviations: MVD, microvessel density; P, paracarcinomatous tissue; T, hepatocellular carcinoma tumor tissue; VEGF, vascular endothelial growth factor; HE, hematoxylin-easin staining. 
Table 2 Association of ADAMTS5 expression with VEGF level and microvessel density in hepatocellular carcinoma tissues

\begin{tabular}{lllll}
\hline & ADAMTS5 & VEGF & CD34 & Tumor size \\
\hline ADAMTS5 & 1.000 & -0.640 & -0.530 & $-0.36 \mathrm{I}$ \\
$P$-value & 0.000 & $<0.001$ & $<0.00 \mathrm{I}$ & 0.012 \\
VEGF & -0.640 & 1.000 & 0.784 & 0.260 \\
$P$-value & $<0.001$ & 0.000 & $<0.001$ & 0.074 \\
CD34 & -0.530 & 0.784 & 1.000 & 0.191 \\
$P$-value & $<0.001$ & $<0.001$ & 0.000 & 0.194 \\
Tumor size & $-0.36 \mathrm{I}$ & 0.260 & 0.191 & 1.000 \\
$P$-value & 0.012 & 0.074 & 0.194 & 0.000 \\
\hline
\end{tabular}

Abbreviation: VEGF, vascular endothelial growth factor.

\section{Discussion}

Previous studies demonstrated that ADAMTS5 protein was aberrantly expressed in several types of human cancer. . $^{8,911,22}$ For example, in prostate cancer, expression of ADAMTS5 mRNA was reduced in tumor tissues and TGF- $\beta$-treated prostatic stromal cells. ${ }^{23}$ The ADAMTS5 promoter was hypermethylated in tissues obtained from colorectal cancer patients when compared to normal mucosae. ${ }^{8}$ Furthermore,
ADAMTS5 functions as an anti-tumorigenic protein independent of its proteoglycanase activity in melanoma cells. ${ }^{21}$ Given these collective observations in separate cancer types, ADAMTS5 may emerge as a putative tumor suppressor gene. Thus, in the current study, we first assessed the expression of ADAMTS5 expression in liver cell lines. We found that the ADAMTS5 $120 \mathrm{kDa}$ zymogen was the major form in liver cell lines, which was consistent with a previous study. ${ }^{3}$ The differential expression levels of ADAMTS5 in normal liver cells and $\mathrm{HCC}$ cell lines indicates a potentially inhibitory role of ADAMTS5 in HCC progression. These are consistent with the status of ADAMTS5 expression observed in prostate cancer and breast cancers. ${ }^{7,23}$

Indeed, tumor microenvironment plays a pivotal role in angiogenesis. Tumor endothelial cells, as a constituent element of tumor microenvironment, could participate in angiogenic signaling via proangiogenic factors (like VEGF) secreted by tumor cells. ${ }^{24}$ In the present study, HCC cell line with stable ADAMTS5 expression was able to downregulate
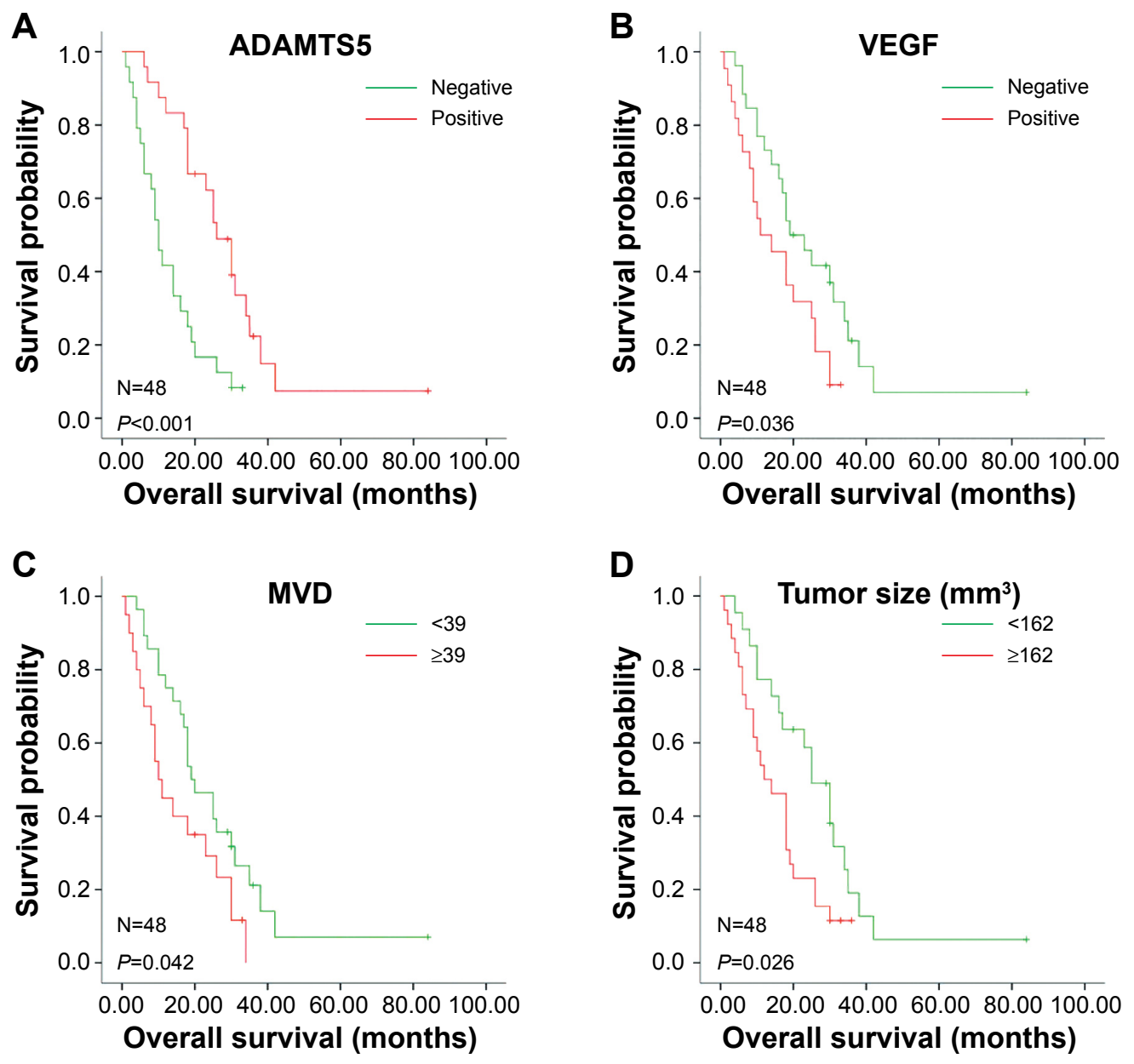

Figure 3 Kaplan-Meier curve analysis of overall survival of patients with HCC.

Notes: (A) Patients with high ADAMTS5-expressed HCC showed better overall survival rates than those with low ADAMTS5-expressed HCC. (B-D) Similarly, patients with relative high levels of VEGF, MVD or tumor size showed poor overall survival rates.

Abbreviations: HCC, hepatocellular carcinoma; MVD, microvessel density; VEGF, vascular endothelial growth factor. 
Table 3 Univariate and multivariate analyses of prognostic factors in 48 patients with hepatocellular carcinoma for overall survival

\begin{tabular}{|c|c|c|c|c|c|c|}
\hline & \multicolumn{3}{|c|}{ Univariate analysis } & \multicolumn{3}{|c|}{ Multivariate analysis } \\
\hline & HR & $95 \% \mathrm{Cl}$ & $\mathbf{P}$ & HR & $95 \% \mathrm{Cl}$ & $\mathbf{P}$ \\
\hline \multicolumn{7}{|l|}{ Sex } \\
\hline Male versus female (43 versus 5 ) & 1.681 & $0.593-4.766$ & 0.329 & & & \\
\hline \multicolumn{7}{|l|}{ Age (years) } \\
\hline$\geq 60$ versus $<60$ ( 16 versus 32$)$ & 0.819 & $0.429-1.560$ & 0.543 & & & \\
\hline \multicolumn{7}{|l|}{ Differentiation } \\
\hline Poor and moderate versus well (42 versus 6 ) & 0.762 & $0.318-1.826$ & 0.542 & & & \\
\hline \multicolumn{7}{|l|}{ Stage } \\
\hline$I+I I$ versus III+IV (26 versus 22$)$ & 0.468 & $0.244-0.901$ & 0.023 & 0.912 & $0.359-2.317$ & 0.847 \\
\hline \multicolumn{7}{|l|}{$\mathrm{HBV}$} \\
\hline Negative versus positive ( 10 versus 38 ) & 0.829 & $0.394-1.747$ & 0.623 & & & \\
\hline \multicolumn{7}{|l|}{$\operatorname{AFP}(n g / m L)$} \\
\hline$<20$ versus $\geq 20$ ( 16 versus 32$)$ & 0.683 & $0.35 \mathrm{I}-1.330$ & 0.262 & & & \\
\hline \multicolumn{7}{|l|}{ ADAMTS5 } \\
\hline Positive versus negative (24 versus 24$)$ & 0.323 & $0.165-0.633$ & 0.001 & 0.308 & $0.115-0.826$ & 0.019 \\
\hline \multicolumn{7}{|l|}{ VEGF } \\
\hline Positive versus negative (22 versus 26 ) & 1.954 & $1.018-3.750$ & 0.044 & 0.632 & $0.213-1.877$ & 0.409 \\
\hline \multicolumn{7}{|l|}{ MVD (counts) } \\
\hline$\geq 39$ versus $<39$ (20 versus 28$)$ & 1.896 & $0.999-3.597$ & 0.050 & 1.767 & $0.845-3.697$ & 0.131 \\
\hline \multicolumn{7}{|l|}{ Tumor size $\left(\mathrm{mm}^{3}\right)$} \\
\hline$\geq 162$ versus $<162$ (26 versus 22$)$ & 2.034 & $1.060-3.901$ & 0.033 & $\mathrm{I} .147$ & $0.436-3.020$ & 0.781 \\
\hline
\end{tabular}

Abbreviations: $\mathrm{Cl}$, confidence interval; HBV, hepatitis B virus; HR, hazard ratio; MVD, microvessel density; VEGF, vascular endothelial growth factor.

VEGF levels in their conditioned medium, which in turn suppressed HUVECs in vitro. Subsequently, a Transwell migration and Matrigel tube formation assay indicated that ADAMTS5 negatively regulates the capacity of tumor cells to promote HUVEC migration and capillary tube formation through downregulating expression of VEGF in vitro. Tumor xenograft assay further showed that ADAMTS5 expression significantly inhibited tumor growth and angiogenesis in vivo by downregulation of VEGF expression. Our results are consistent with the function of ADAMTS5 in B16 melanoma cells and imply an antiangiogenic role of ADAMTS5 in HCC, making ADAMTS5 a promising molecular target for HCC therapy.

To our knowledge, this is the first study of its kind to investigate the association between ADAMTS5 expression and HCC patients prognosis. By analyzing the association between ADAMTS5 expression and clinicopathological characteristics, it has been found that the larger tumor size in patients correlates with low ADAMTS5 expression compared with high ADAMTS5 expression. Results of Kaplan-Meier survival analysis curves showed that patients with high ADAMTS5-expressed HCC had better OS than the patients without or with low expression of ADAMTS5. In contrast, VEGF expression, MVD, and tumor size showed the opposite result. However, the multivariate analysis showed that only ADAMTS5 expression was an independent prognostic factor for HCC patients. Further studies are needed to verify our current data. Our results suggest that high ADAMTS5 expression is associated with a good outcome in HCC, and may be used as a prognostic marker.

The expression and contribution of ADAMTS5 vary in different types of cancer and the expression is regulated by different transcription factors. ${ }^{10,21,23}$ A previous study showed that ADAMTS5 can be transcriptionally regulated by $\mathrm{NF}-\mathrm{\kappa B}$ in chondrocytes during osteoarthritis development. ${ }^{25}$ The decreased expression of ADAMTS5 in HCC may be the result of a joint action of multiple transcription factors (TFs) during HCC progression. Furthermore, our current data demonstrated that ADAMTS5 inhibited angiogenesis by downregulation of VEGF expression, although it remains unknown what the underlying molecular mechanism is by which ADAMTS5 regulates VEGF. Previous studies showed that members of the ADAMTS family such as ADAMTS1 and ADAMTS4 could inhibit angiogenesis through direct binding to the $V E G F$, which may provide a direction for future investigation. ${ }^{26,27}$ Our current study is a proof of principle and the small sample size in our study is a limitation. Thus, the role of ADAMTS5 in HCC need to be further investigated. 
A

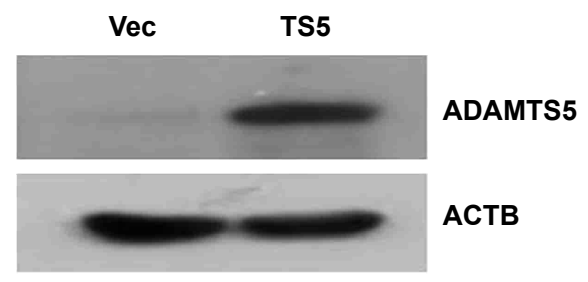

C

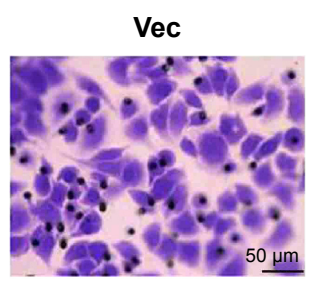

TS5 + PBS

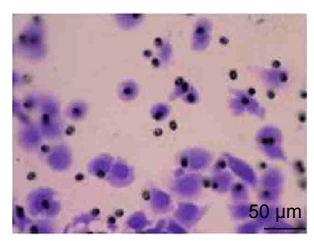

Vec

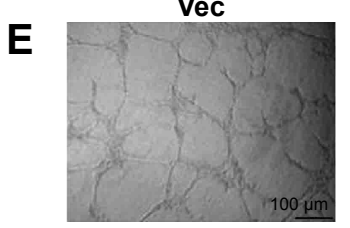

TS5 + PBS

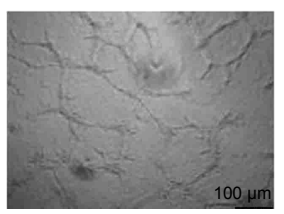

TS5

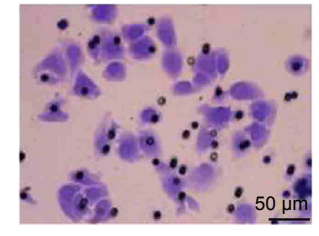

TS5 + VEGF

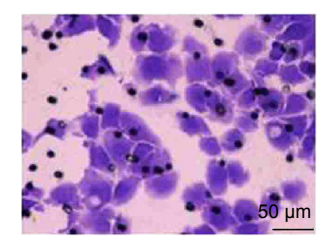

TS5

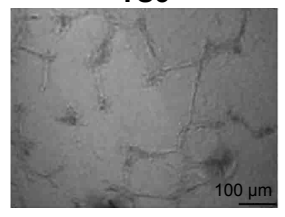

TS5 + VEGF

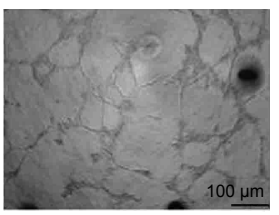

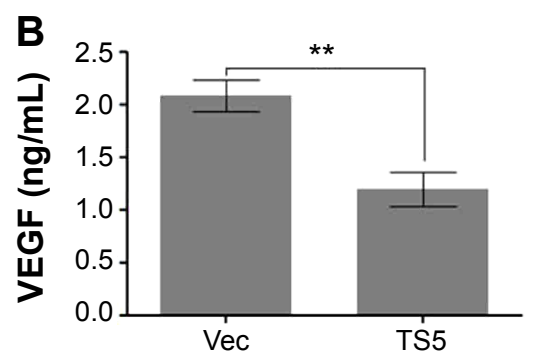

D
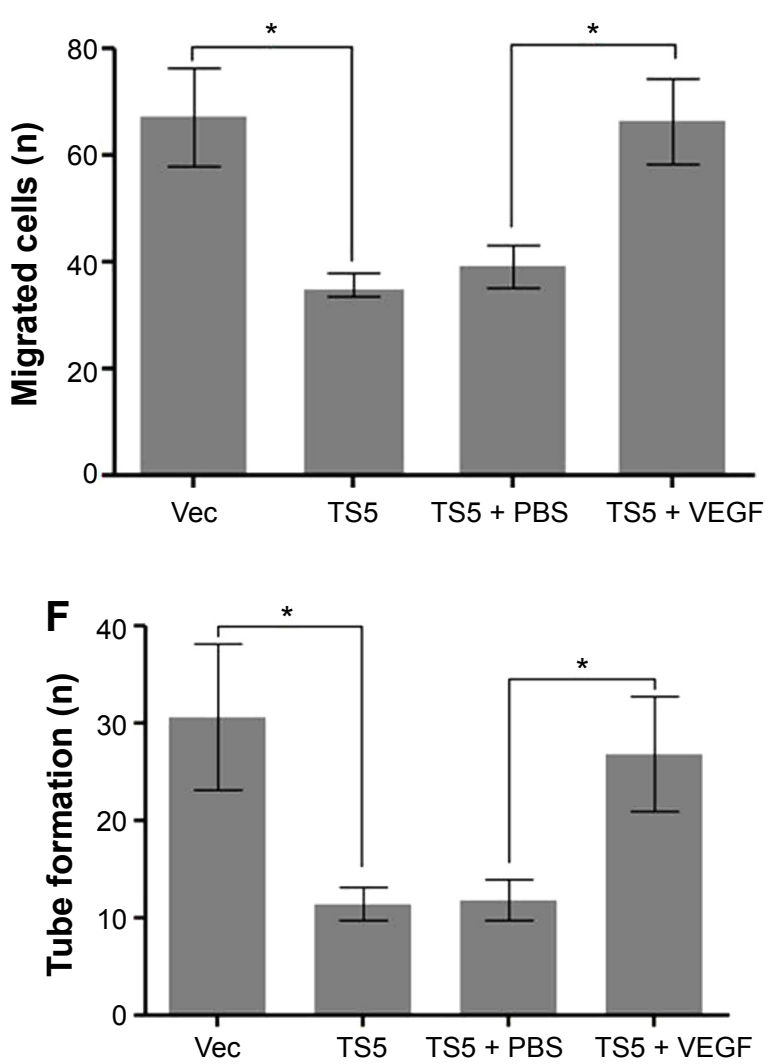

Figure 4 Inhibitory effect of ADAMTS5-expressed hepatocellular carcinoma cell-conditioned medium on HUVEC migration and tube formation.

Notes: (A) Western blot. Hep3B cells were stably transfected with empty vector (Vec group) or full-length ADAMTS5 (TS5 group) and subjected to Western blot analysis of ADAMTS5 expression level. (B) ELISA. Tumor cell culture conditioned medium was assessed for levels of VEGF by ELISA. Columns, mean ( $n=3$ ); bars, SEM. $* * P<0.00$ I compared to controls. (C) Transwell migration assay. HUVECs were grown and treated with tumor cell culture conditioned medium and then subjected to Transwell migration assay. Representative images of migration assay are presented. (D) Quantitative analysis of HUVEC migration. The graph shows the summarized data. Columns, mean $(n=5)$; bars, SEM. $* P<0.0$ I compared to controls. (E) HUVECs were cultured in 24-well plates coated with Matrigel and then treated with tumor cell-conditioned medium derived from Hep3B cells stably transfected with empty vector or full-length ADAMTS5. Representative images of tube formation assay are presented. (F) The graph shows the quantified data of HUVEC angiogenesis. Columns, mean $(n=5)$; bars, SEM. $* P<0.01$ compared to controls.

Abbreviations: ELISA, enzyme-linked immunosorbent assay; HUVEC, human umbilical vein endothelial cell; PBS, phosphate-buffered saline; SEM, standard error of the mean; VEGF, vascular endothelial growth factor.

\section{Conclusion}

Our study suggests that ADAMTS5 is downregulated in HCC cell lines than in their benign counterparts. ADAMTS5 inhibits tumor growth by suppressing angiogenesis in HCC. The antiangiogenic function of ADAMTS5 in HCC can be mediated through downregulating VEGF. High expression of ADAMTS5 correlated with good prognosis of HCC. Our findings indicate that ADAMTS5 may represent a promising novel therapeutic target and a potential prognostic marker in HCC.

\section{Acknowledgments}

We thank SH Zhang for her help in carrying out clinical statistics. We also thank Medjaden Bioscience Limited for their 
A

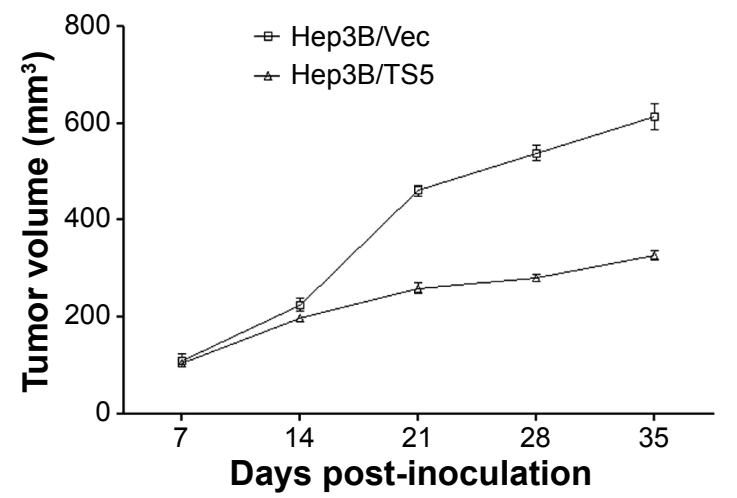

C
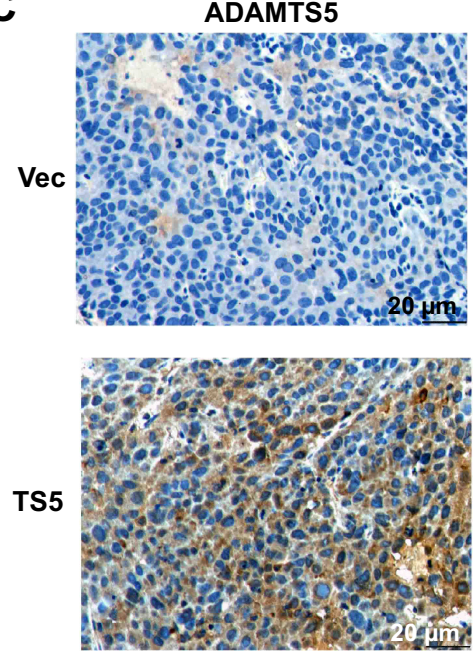

B

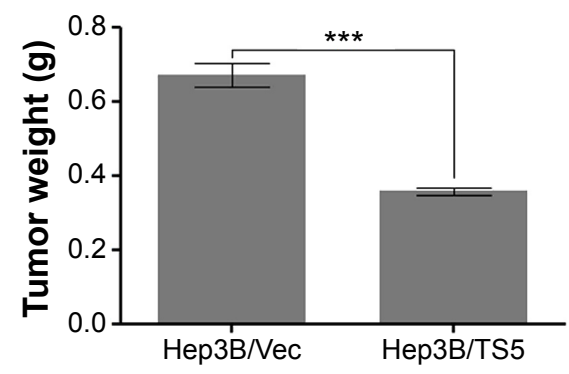

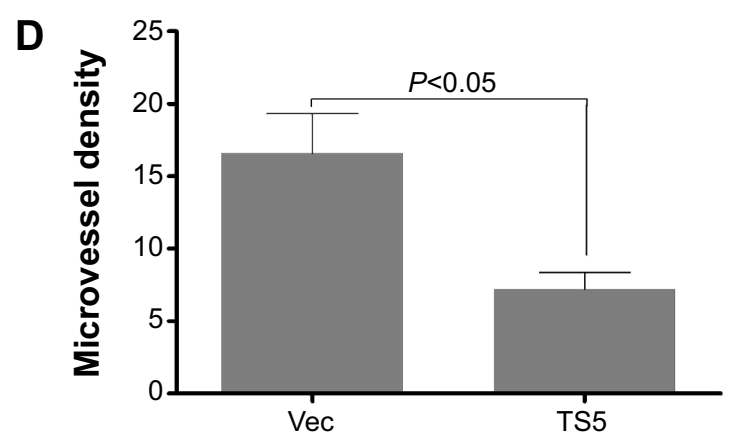

Figure 5 Overexpression of ADAMTS5 inhibits hepatocellular carcinoma cell xenograft growth and angiogenesis in a mouse xenograft model.

Notes: An in vivo hepatoma model was established by injection of $1 \times 10^{7} \mathrm{Hep3B} / \mathrm{TS} 5$ or Hep3B/Vec cells. (A) Tumor xenograft growth curve. Comparison of tumor growth (volume increase) between ADAMTS5 group (Hep3B/TS5) and the control group (Hep3B/Vec). The volume of tumor xenografts was monitored every 7 days and measured using vernier calipers. The tumor volume $(V)$ was calculated using the formula of $V=A \times B^{2} \times 0.52$, where $A$ was the largest diameter (mm) and $B$ was the smallest diameter $(\mathrm{mm})(\mathrm{n}=3 /$ group). (B) The tumors were weighed immediately after isolation from mice. The tumor weight was plotted between the two groups. Columns, mean ( $\mathrm{n}=3$ ); bars, standard error of the mean. $* * * P<0.0001$ compared to controls. (C) Representative photographs of immunohistochemical analysis of ADAMTS5, VEGF, and CD34 expression in xenograft sections from mice (original magnification, 400x). (D) Summarized data show that ADAMTS5 decreased the number of CD34-positive vessels in xenograft sections. The number of vessels was counted in five fields for each xenograft section. The data are presented as means \pm standard deviation.

Abbreviation: VEGF, vascular endothelial growth factor.

assistance in the preparation of this manuscript. This work was supported by the National Natural Science Foundation of China (number 30872975 and number 81270482).

\section{Disclosure}

The authors report no conflicts of interest in this work.

\section{References}

1. Jemal A, Bray F, Center MM, Ferlay J, Ward E, Forman D. Global cancer statistics. CA Cancer J Clin. 2011;61(2):69-90.

2. Forner A, Llovet JM, Bruix J. Hepatocellular carcinoma. Lancet. 2012;379(9822):1245-1255.

3. Turner SL, Mangnall D, Bird NC, Bunning RA, Blair-Zajdel ME. Expression of ADAMTS-1, ADAMTS-4, ADAMTS-5 and TIMP3 by hepatocellular carcinoma cell lines. Int J Oncol. 2012;41(3):1043-1049. 
4. Kintakas C, McCulloch DR. Emerging roles for ADAMTS5 during development and disease. Matrix Biol. 2011;30(5-6):311-317.

5. Glasson SS, Askew R, Sheppard B, et al. Deletion of active ADAMTS5 prevents cartilage degradation in a murine model of osteoarthritis Nature. 2005;434(7033):644-648.

6. Stanton H, Rogerson FM, East CJ, et al. ADAMTS5 is the major aggrecanase in mouse cartilage in vivo and in vitro. Nature. 2005; 434(7033):648-652.

7. Porter S, Scott SD, Sassoon EM, et al. Dysregulated expression of adamalysin-thrombospondin genes in human breast carcinoma. Clin Cancer Res. 2004;10(7):2429-2440.

8. Kim YH, Lee HC, Kim SY, et al. Epigenomic analysis of aberrantly methylated genes in colorectal cancer identifies genes commonly affected by epigenetic alterations. Ann Surg Oncol. 2011;18(8):2338-2347.

9. Roman-Gomez J, Jimenez-Velasco A, Agirre X, Prosper F, Heiniger A, Torres A. Lack of $\mathrm{CpG}$ island methylator phenotype defines a clinical subtype of T-cell acute lymphoblastic leukemia associated with good prognosis. J Clin Oncol. 2005;23(28):7043-7049.

10. Nakada M, Miyamori H, Kita D, et al. Human glioblastomas overexpress ADAMTS-5 that degrades brevican. Acta Neuropathol. 2005;110(3):239-246.

11. Held-Feindt J, Paredes EB, Blömer U, et al. Matrix-degrading proteases ADAMTS4 and ADAMTS5 (disintegrins and metalloproteinases with thrombospondin motifs 4 and 5) are expressed in human glioblastomas. Int $J$ Cancer. 2006;118(1):55-61.

12. Xu B, Cai Z, Zeng Y, et al. $\alpha$-Methylacyl-CoA racemase (AMACR) serves as a prognostic biomarker for the early recurrence/metastasis of HCC. J Clin Pathol. 2014;67(11):974-979.

13. Wang D, Zhong ZY, Li MX, Xiang DB, Li ZP. Vector-based Ape1 small interfering RNA enhances the sensitivity of human osteosarcoma cells to endostatin in vivo. Cancer Sci. 2007;98(12):1993-2001.

14. Li Y, Tang Y, Ye L, et al. Establishment of a hepatocellular carcinoma cell line with unique metastatic characteristics through in vivo selection and screening for metastasis-related genes through cDNA microarray. $J$ Cancer Res Clin Oncol. 2003;129(1):43-51.

15. Chen J, Liu WB, Jia WD, et al. Embryonic morphogen nodal is associated with progression and poor prognosis of hepatocellular carcinoma. PLoS One. 2014;9(1):e85840.

16. Lai KB, Sanderson JE, Yu CM. The regulatory effect of norepinephrine on connective tissue growth factor (CTGF) and vascular endothelial growth factor (VEGF) expression in cultured cardiac fibroblasts. Int $J$ Cardiol. 2013;163(2):183-189.
17. Sharghi-Namini S, Fan H, Sulochana KN, et al. The first but not the second thrombospondin type 1 repeat of ADAMTS5 functions as an angiogenesis inhibitor. Biochem Biophys Res Commun. 2008; 371(2):215-219.

18. King ML, Lindberg ME, Stodden GR, et al. WNT7A/ $\beta$-catenin signaling induces FGF1 and influences sensitivity to niclosamide in ovarian cancer. Oncogene. Epub 2014 Sep 1.

19. Jin H, Lv S, Yang J, et al. Use of microRNA Let-7 to control the replication specificity of oncolytic adenovirus in hepatocellular carcinoma cells. PLoS One. 2011;6(7):e21307.

20. Zhou B, Ma R, Si W, et al. MicroRNA-503 targets FGF2 and VEGFA and inhibits tumor angiogenesis and growth. Cancer Lett. 2013; 333(2):159-169.

21. Kumar S, Sharghi-Namini S, Rao N, Ge R. ADAMTS5 functions as an anti-angiogenic and anti-tumorigenic protein independent of its proteoglycanase activity. Am J Pathol. 2012;181(3):1056-1068.

22. Sjöblom T, Jones S, Wood LD, et al. The consensus coding sequences of human breast and colorectal cancers. Science. 2006; 314(5797):268-274.

23. Cross NA, Chandrasekharan S, Jokonya N, et al. The expression and regulation of ADAMTS- $1,-4,-5,-9$, and -15 , and TIMP- 3 by TGFbeta 1 in prostate cells: relevance to the accumulation of versican. Prostate. 2005;63(3):269-275.

24. Mittal K, Ebos J, Rini B. Angiogenesis and the tumor microenvironment: vascular endothelial growth factor and beyond. Semin Oncol. 2014;41(2):235-251.

25. Kobayashi H, Hirata M, Saito T, Itoh S, Chung UI, Kawaguchi H. Transcriptional induction of ADAMTS5 protein by nuclear factor- $\mathrm{\kappa B}$ (NF-KB) family member RelA/p65 in chondrocytes during osteoarthritis development. J Biol Chem. 2013;288(40):28620-28629.

26. Kahn J, Mehraban F, Ingle G, Xin X, Bryant JE, Vehar G et al. Gene expression profiling in an in vitro model of angiogenesis. Am J Pathol. 2000;156(6):1887-1900.

27. Luque A, Carpizo DR, Iruela-Arispe ML. ADAMTS1/METH1 inhibits endothelial cell proliferation by direct binding and sequestration of VEGF165. J Biol Chem. 2003;278(26):23656-23665.

\section{Publish your work in this journal}

Drug Design, Development and Therapy is an international, peerreviewed open-access journal that spans the spectrum of drug design and development through to clinical applications. Clinical outcomes, patient safety, and programs for the development and effective, safe, and sustained use of medicines are a feature of the journal, which

\section{Dovepress}

has also been accepted for indexing on PubMed Central. The manuscript management system is completely online and includes a very quick and fair peer-review system, which is all easy to use. Visit http://www.dovepress.com/testimonials.php to read real quotes from published authors. 